\title{
Single-Mode Operation of a Coiled Multimode Fiber Amplifier
}

\author{
Jeffrey P. Koplow \\ Naval Research Laboratory, Washington, DC 20375 \\ Dahv A.V. Kliner \\ Sandia National Laboratories, Livermore, CA 94551 \\ Lew Goldberg \\ Naval Research Laboratory, Washington, DC 20375 \\ RECEIVED \\ FEB 142000 \\ OSTI
}

\begin{abstract} quality.

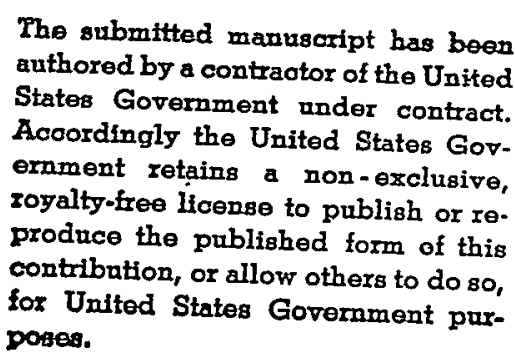

We report a new approach to obtain single-transverse-mode operation of a multimode fiber amplifier, in which the gain fiber is coiled to induce significant bend loss for all but the lowestorder mode. We have demonstrated this method by constructing a coiled amplifier using $\mathrm{Yb}-$ doped, double-clad fiber with a core diameter of $25 \mu \mathrm{m}$ and NA of $\sim 0.1(\mathrm{~V} \approx 7.4)$. When operated as an ASE source, the output beam had an $M^{2}$ value of $1.09 \pm 0.09$; when seeded at 1064 $\mathrm{nm}$, the slope efficiency was similar to that of an uncoiled amplifier. This technique does not require exotic fiber designs or increase system complexity and is inexpensive to implement. It will allow scaling of pulsed fiber lasers and amplifiers to significantly higher pulse energies and peak powers and cw fiber sources to higher average powers while maintaining excellent beam 
Single-mode (SM), rare-earth-doped fiber lasers and amplifiers are finding widespread use in applications requiring compact, rugged optical sources with diffraction-limited beam quality. The advent of double-clad fibers has allowed these sources to be scaled to average powers of $>100 \mathrm{~W} .{ }^{1}$ For applications requiring high-energy pulses (e.g., nonlinear frequency conversion, ${ }^{2}$ pumping of optical parametric oscillators, ${ }^{3}$ lidar, materials processing), however, use of fiberbased systems has been limited by the relatively low pulse energies available compared to bulk lasers. This pulse-energy limitation arises from two factors: low energy storage and the onset of nonlinear processes in the fiber. The energy-storage capacity of a fiber is determined by amplified spontaneous emission (ASE), which limits the maximum population inversion. ${ }^{4}$ The most important nonlinear processes that limit the pulse energy and peak power are stimulated Raman scattering (SRS) and stimulated Brillouin scattering (SBS); the relative importance of these processes is determined by the pulse duration, spectral linewidth, and fiber length. ${ }^{5}$ For $\mathrm{cw}$ applications requiring narrow linewidth, the maximum power is limited by SBS.

Two approaches to overcoming these limitations have been reported. Taverner et al. developed large-mode-area, Er-doped SM fibers with NA's of $0.066-0.08$ and core diameters of 14-17 $\mu \mathrm{m} .{ }^{6,7}$ Decreasing the NA (relative to standard telecommunication values of $\sim 0.15$ ) allows the core size to be increased while maintaining SM operation. The resultant increased mode-field area raises the threshold for nonlinear processes. In addition, the lower NA reduces the fraction of spontaneous emission captured by the fiber, thereby increasing energy storage. ${ }^{8}$ Several groups have used multimode (MM) fiber amplifiers and have obtained varying levels of suppression of high-order modes by adjusting the fiber index and dopant distributions, ${ }^{9,10}$ cavity configurations, ${ }^{11,12}$ and/or launch conditions of the seed beam. ${ }^{13-17}$ In all of these approaches, increasing the rare-earth-dopant concentration (i.e., the pump absorption coefficient) allows the use of shorter fibers, which proportionally increases the threshold power for SRS and SBS.

In this paper, we report a new approach to obtain SM operation of a laser or amplifier employing MM fiber. By wrapping the gain fiber around a cylindrical mandrel whose radius is chosen to provide low loss for the fundamental mode $\left(\mathrm{LP}_{01}\right)$ and high loss for $\mathrm{LP}_{11}$ and the other 


\section{DISCLAIMER}

This report was prepared as an account of work sponsored by an agency of the United States Government. Neither the United States Government nor any agency thereof, nor any of their employees, make any warranty, express or implied, or assumes any legal liability or responsibility for the accuracy, completeness, or usefulness of any information, apparatus, product, or process disclosed, or represents that its use would not infringe privately owned rights. Reference herein to any specific commercial product, process, or service by trade name, trademark, manufacturer, or otherwise does not necessarily constitute or imply its endorsement, recommendation, or favoring by the United States Government or any agency thereof. The views and opinions of authors expressed herein do not necessarily state or reflect those of the United States Government or any agency thereof. 


\section{DISCLAIMER}

Portions of this document may be illegible in electronic image products. Images are produced from the best available original document. 
high-order modes, bend loss can be used as a form of distributed spatial filtering. This technique exploits the fact that $\mathrm{LP}_{01}$ is the least sensitive to bend loss and that, for all modes, the bend-loss attenuation coefficient $(\alpha, \mathrm{dB} / \mathrm{m})$ depends exponentially on the radius of curvature. ${ }^{18}$ As in the case of SM fibers, the stored energy and peak-power-handling capability are maximized by making the NA as low as possible and the concentration of the rare-earth dopant as high as possible. Exploiting bend loss to discriminate against higher-order modes, however, allows the core diameter to be increased significantly beyond the SM limit (i.e., the restriction $V<2.405$ is eliminated), thereby allowing fiber lasers and amplifiers to be scaled up to much higher peak and average powers. Furthermore, for double-clad fiber with a given inner-cladding area, the pump absorption coefficient increases quadratically with core diameter, permitting the use of shorter lengths of fiber; alternatively, increasing both the core and inner-cladding sizes allows the use of larger, higher-power pump sources without necessitating prohibitively long fibers. Although this approach is fully compatible with (and may be extended by) other methods for suppressing modes above $\mathrm{LP}_{01}$, it does not require that the launch conditions and mode quality of the seed beam be carefully matched to $\mathrm{LP}_{01}$, nor does it require exotic fiber designs to obtain diffractionlimited performance.

The mode-filtering effect that is the basis of the coiled amplifier is shown in Fig. 1. Following the analysis of Marcuse ${ }^{18}$, we calculated the attenuation for $\operatorname{LP}_{11}\left(\gamma_{11}=\alpha_{11} L\right.$, where $L$ is the fiber length) as a function of fiber core diameter for specified values of $\mathrm{LP}_{01}$ attenuation $\left(\gamma_{01}\right)$. This calculation applies to a step-index fiber with an NA of 0.08 (a typical value for a low$N A$ fiber) and $L=10 \mathrm{~m}$. The attenuation coefficients for modes of order higher than $L P_{11}$ are larger than $\alpha_{11}$, and these modes will therefore be even more effectively suppressed in the coiled amplifier. Figure 1 indicates that, depending on the acceptable level of $\mathrm{LP}_{01}$ bend loss, $>10 \mathrm{~dB}$ of suppression for the higher-order modes is possible for core diameters of $50-100 \mu \mathrm{m}(\mathrm{V}$ numbers of 12-24). The corresponding bend radii are calculated to be $1.7-3.7 \mathrm{~cm}$, which is compatible with compact packaging of the amplifier. This calculation neglects the effect of field deformation caused by fiber curvature, which decreases $\alpha_{01}$ relative to the attenuation 
coefficients of the higher-order modes, ${ }^{19}$ i.e., the calculation provides a conservative estimate of $\gamma_{11}$ relative to $\gamma_{01}$. Furthermore, for a rare-earth-doped fiber, additional suppression of $L_{11}$ relative to $\mathrm{LP}_{01}$ is provided by the spatial overlap of the mode field distributions with the gain region (particularly at lower $\mathrm{V}$ numbers). ${ }^{20}$ Finally, the output power of a coiled amplifier will be reduced by less than $\gamma_{01}$ because most of the extracted power does not travel the entire length of the amplifier (especially when the signal and pump beams are counterpropagating).

We tested the performance of a mode-filtered amplifier using $6 \mathrm{~m}$ of $\mathrm{Yb}$-doped, double-clad fiber with a core diameter of $25 \mu \mathrm{m}$, core NA of $\sim 0.1(\mathrm{~V} \approx 7.4$ at $1064 \mathrm{~nm})$, and $\mathrm{Yb}$ concentration of $\sim 1.5 \mathrm{wt} . \%$. The $200-\mu \mathrm{m}$-diameter hexagonal inner cladding had an NA of 0.47 , and the absorption coefficient at $975 \mathrm{~nm}$ was $\sim 7.5 \mathrm{~dB} / \mathrm{m}$. The fiber was end pumped by imaging the output of a $\sim 974-\mathrm{nm}$, fiber-coupled $(250-\mu \mathrm{m}$ diameter, $\mathrm{NA}=0.22)$ diode array with a maximum output power of $8.2 \mathrm{~W}$ (OptoPower) onto the face of the fiber. The launched pump power $\left(P_{\text {pump }}\right)$ was measured to be $89 \%$ of the power emitted by the pump fiber (using a short piece of double-clad fiber). For some experiments, the amplifier was seeded at $1064 \mathrm{~nm}$ by a single-longitudinal-mode Nd:YAG laser (Lightwave Series 122) using a dichroic beamsplitter to combine the pump and seed beams in a copropagating geometry. The output end of the fiber amplifier was mode-stripped to remove ASE and residual pump light propagating in the inner cladding. ${ }^{21}$ Both ends were connectorized and angle polished at $8^{\circ}$.

We first measured the ASE power and near-field spatial profile of the amplifier as a function of spool diameter $(D)$ to verify the efficacy of using bend loss to obtain SM operation of the MM amplifier and to determine the optimum spool diameter. For these experiments, the gain fiber was pumped with $3.9 \mathrm{~W}$, and the copropagating ASE from the output end of the amplifier was imaged onto a 2048-element linear diode array (EG\&G Reticon) using a 5.25-mm focal length microscope objective (magnification $\approx 550$ ). The results, which are summarized in Fig. 2 , demonstrate the suppression of higher-order modes before the onset of significant loss for $\mathrm{LP}_{01}$. From these measurements, we chose $D=1.58 \mathrm{~cm}$ to construct the coiled amplifier. 
The two helical polarities of the $\mathrm{LP}_{11}$ mode have intensity distributions whose maxima are separated by a $90^{\circ}$ rotation about the fiber axis. ${ }^{20}$ A single coil will have higher loss for the $L P_{11}$ polarity with intensity maxima in the plane of curvature. Rather than decrease $D$ to increase the loss for the other $\mathrm{LP}_{11}$ mode (which would also increase $\gamma_{01}$ ), we obtained optimum suppression of $\mathrm{LP}_{11}$ relative to $\mathrm{LP}_{01}$ by using two spools of equal diameter oriented orthogonally, each wrapped with $3 \mathrm{~m}$ of the gain fiber. Bend-loss discrimination between the two helical polarities of the $\mathrm{LP}_{11}$ mode (and between other high-order modes) may account for the elliptical beams observed in other MM amplifiers. ${ }^{9}$

Figure 3a shows a measurement of $\mathrm{M}^{2}$ for the coiled amplifier with $D=1.58 \mathrm{~cm} .{ }^{22}$ The bestfit value of $\mathrm{M}^{2}$ was $1.09 \pm 0.09$ (two standard deviations), confirming SM operation of the coiled $\mathrm{MM}$ amplifier.

Because spontaneous emission uniformly excites all modes of the fiber, the measurements shown in Figs. 2 and 3a constitute a stringent test of the use of bend loss for constructing a mode-filtered amplifier. In many applications, the cavity configuration or launch condition of the seed beam can provide further suppression of high-order modes..$^{11-13,15}$

We measured the saturated output power as a function of pump power for both the uncoiled and coiled amplifier by seeding with $\sim 40 \mathrm{~mW}$ at $1064 \mathrm{~nm}$ and varying the current to the pump diode. As shown in Fig. 3b, coiling the amplifier did not significantly degrade the performance. The nearly unchanged slope efficiency and small $(\sim 30 \%)$ increase in threshold are consistent with the introduction of some bend loss for $\mathrm{LP}_{01}$. These data demonstrate that $\gamma_{01}$ was not prohibitively large (in agreement with Fig. 2) and that mode conversion from $\mathrm{LP}_{01}$ to higherorder modes was not significant (in accord with previous results). ${ }^{13,15}$ In experiments with a higher-power pump laser, a saturated output power of $4.1 \mathrm{~W}$ from the coiled amplifier was obtained with $P_{\text {pump }}=11 \mathrm{~W}$. The spatial profile of the output beam from the seeded, coiled amplifier was similar to that shown in Fig. 2 (inset) for ASE with $D=1.58 \mathrm{~cm}$.

In conclusion, the present technique will allow scaling of pulsed fiber lasers and amplifiers to significantly higher pulse energies, peak powers, and average powers (and narrow-linewidth $\mathrm{cw}$ 
lasers to higher average powers) while maintaining diffraction-limited beam quality. The larger mode-field diameter and shorter fiber length of a MM amplifier provide a higher threshold power for the onset of nonlinear processes (SBS, SRS, and self-phase modulation ${ }^{23}$ ); for Er-doped double-clad fibers, the larger pump absorption coefficient may eliminate the need for $\mathrm{Yb}$ codoping. The MM fiber used in the proof-of-concept experiments reported here does not represent the limit of this approach; calculations indicate that core diameters of $>50 \mu \mathrm{m}$ will be possible for low-NA fibers. As indicated in Fig. 1, however, increasing the core diameter reduces the discrimination between $\mathrm{LP}_{01}$ and $\mathrm{LP}_{11}$, and the coiled $\mathrm{MM}$ fiber will eventually support more than the fundamental mode. The practical limit on the core size for bend-lossinduced mode-filtering depends on the acceptable levels of high-order mode suppression and $\mathrm{LP}_{01}$ loss for a particular application and needs to be determined empirically for a given fiber. The technique may be used in conjunction with other methods for suppressing high-order modes. For situations in which the mode quality or launch conditions of the seed beam make it difficult to preferentially excite $\mathrm{LP}_{01}$, the input end of the gain fiber can be coiled more tightly than the rest of the amplifier to provide additional discrimination against high-order modes. Finally, the coiled MM amplifier is compatible with the use of tension-coiling and/or bending-induced birefringence for constructing polarization-maintaining fiber amplifiers. ${ }^{24}$

JPK and LG acknowledge support from the Office of Naval Research and the Air Force Philips Laboratory. DAVK acknowledges support from Sandia's LDRD program, NASA (Instrument Incubator Program), and the NSF (ATM-9522028). 


\section{References}

1. V. Dominic, S. MacCormack, R. Waarts, S. Sanders, S. Bicknese, R. Dohle, E. Wolak, P.S. Yeh, and E. Zucker, in Conference on Lasers and Electro-Optics (Optical Society of America, Washington DC, 1999), paper CPD11.

2. J.P. Koplow, D.A.V. Kliner, and L. Goldberg, IEEE Photon. Technol. Lett. 10, 75 (1998).

3. P.E. Britton, H.L. Offerhaus, D.J. Richardson, P.G.R. Smith, G.W. Ross, and D.C. Hanna, Opt. Lett. 24, 975 (1999).

4. J. Nilsson and B. Jaskorzynska, Opt. Lett. 18, 2099 (1993).

5. G.P. Agrawal, Nonlineàr Fiber Optics (Academic Press, San Diego, 1995).

6. D. Taverner, D.J. Richardson, L. Dong, J.E. Caplen, K. Williams, and R.V. Penty, Opt. Lett. 22, 378 (1997).

7. G.P. Lees, D. Taverner, D.J. Richardson, L. Dong, and T.P. Newson, Electron. Lett. 33, 393 (1997).

8. J. Nilsson, R. Paschotta, J.E. Caplen, and D.C. Hanna, Opt. Lett. 22, 1092 (1997).

9. H.L. Offerhaus, N.G. Broderick, D.J. Richardson, R. Sammut, J. Caplen, and L. Dong, Opt. Lett. 23, 1683 (1998).

10. J.M. Sousa and O.G. Okhotnikov, Appl. Phys. Lett. 74, 1528 (1999).

11. U. Griebner, R. Koch, H. Schönnagel, and R. Grunwald, Opt. Lett. 21, 266 (1996).

12. U. Griebner and H. Schönnagel, Opt. Lett. 24, 750 (1999).

13. O.G. Okhotnikov and J.M. Sousa, Electron. Lett. 35, 1011 (1999).

14. C.C. Renaud, R.J. Selvas-Aguilar, J. Nilsson, P.W. Turner, and A.B. Grudinin, IEEE Photon. Technol. Lett. 11, 976 (1999).

15. M.E. Fermann, Opt. Lett. 23, 52 (1998).

16. M. Hofer, M.E. Fermann, A. Galvanauskas, D. Harter, and R.S. Windeler, IEEE Photon. Technol. Lett. 11, 650 (1999).

17. I. Zawischa, K. Plamann, C. Fallnich, H. Welling, H. Zellmer, and A. Tünnermann, Opt. Lett. 24, 469 (1999). 
18. D. Marcuse, J. Opt. Soc. Am. 66, 216 (1976).

19. D. Marcuse, J. Opt. Soc. Am. 66, 311 (1976).

20. C.C. Davis, Lasers and Electro-Optics (Cambridge University Press, Cambridge, 1996).

21. J.P. Koplow, L. Goldberg, and D.A.V. Kliner, IEEE Photon. Technol. Lett. 10, 793 (1998).

22. M.W. Sasnett and T.F. Johnston in Laser Beam Diagnostics, edited by R.N. Hindy and Y. Kohanzadeh (Proc. SPIE 1414, Bellingham, Washington, 1991), p. 21.

23. A. Galvanauskas, D. Harter, M.A. Arbore, M.H. Chou, and M.M. Fejer, Opt. Lett. 23, 1695 (1999).

24. J.P. Koplow, L. Goldberg, R.P. Moeller, and D.A.V. Kliner, Opt. Lett. (in press, 2000). 


\section{Figure Captions}

Figure 1. Calculated values of $\gamma_{11}$ versus core diameter at the indicated values of $\gamma_{01}$. The choice of $L=10 \mathrm{~m}$ is representative; for other fiber lengths, similar levels of $\mathrm{LP}_{11}$ suppression are calculated for a given $\gamma_{01}$ at slightly different values of $D$.

Figure 2. Copropagating ASE power as a function of spool diameter for the MM amplifier with $P_{\text {pump }}=3.9 \mathrm{~W}$. The ASE power for a loosely coiled amplifier $(D>20 \mathrm{~cm})$ was $140 \mathrm{~mW}$. Inset: Near-field intensity distribution of ASE from the MM amplifier for various spool diameters. The curves have been off-set for clarity, and the integrated areas correspond to the ASE powers. FWHM denotes the full-width at half-maximum of the best-fit Gaussian curve for each profile; the fit is shown for $D=1.58 \mathrm{~cm}$ (solid line). Much of the scatter in the data arises from pixel-topixel variations in the sensitivity of the diode array.

Figure 3. (a) Measurement of $\mathrm{M}^{2}$ for copropagating ASE from the coiled $(D=1.58 \mathrm{~cm}) \mathrm{MM}$ amplifier with $P_{\text {pump }}=3.9 \mathrm{~W}$. The ASE was focused with a microscope objective $(f=16 \mathrm{~mm})$, and the $1 / e^{2}$ beam radius $[\omega(z)]$ was measured at various positions $(z)$ near the beam waist $(z=$ 0 ); $\omega(z)$ was measured by chopping the beam at $\sim 100 \mathrm{~Hz}$ and using a photodiode and digitizing oscilloscope to measure the rise time of the chopped beam. The solid curve is the best fit assuming a Gaussian $\left(\mathrm{LP}_{01}\right)$ beam. The dashed curve (nearly indistinguishable from the solid curve) is the best fit to the equation used to determine $M^{2}$ (shown in the legend). ${ }^{22}$ (b) Saturated output power as a function of $P_{\text {pump }}$ for the uncoiled and coiled MM amplifiers seeded at $1064 \mathrm{~nm}$ (copropagating). The curvature at low pump powers resulted from shifting of the pump-laser wavelength as the current was varied. The slope efficiency and threshold were determined from linear least-squares fits to the data with $P_{\text {pump }}>5 \mathrm{~W}$. 
Figure 1

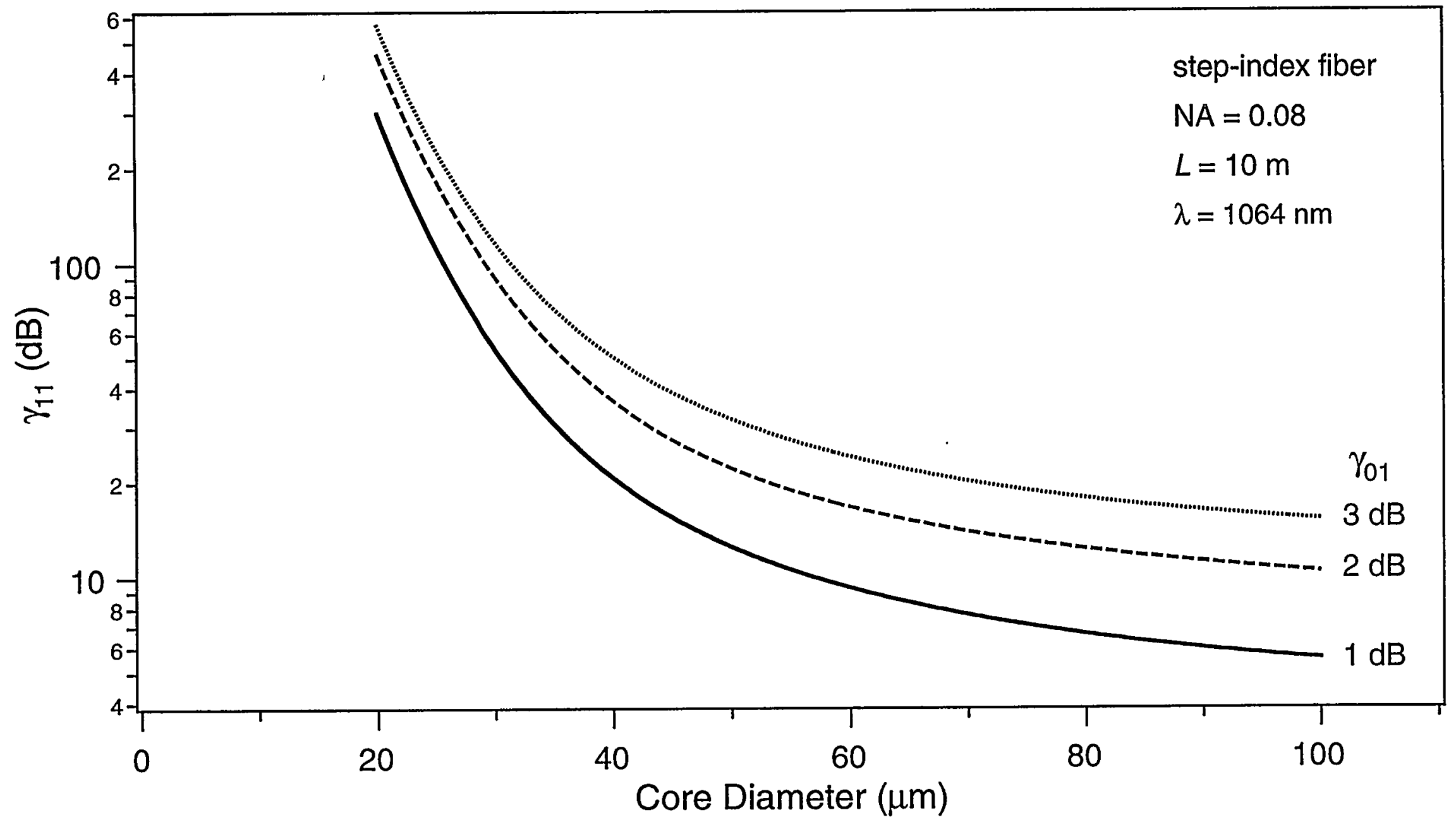


Figure 2

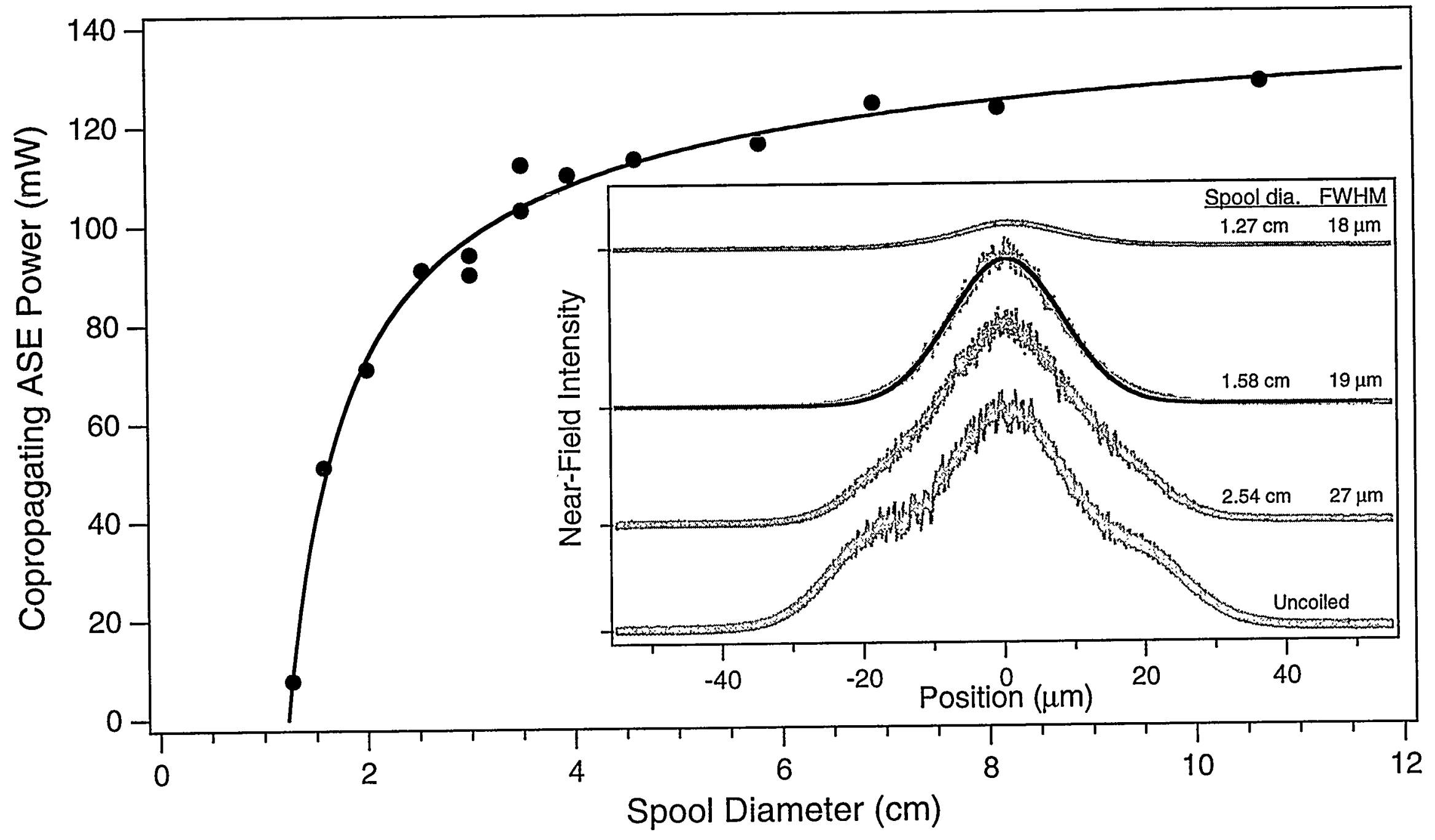




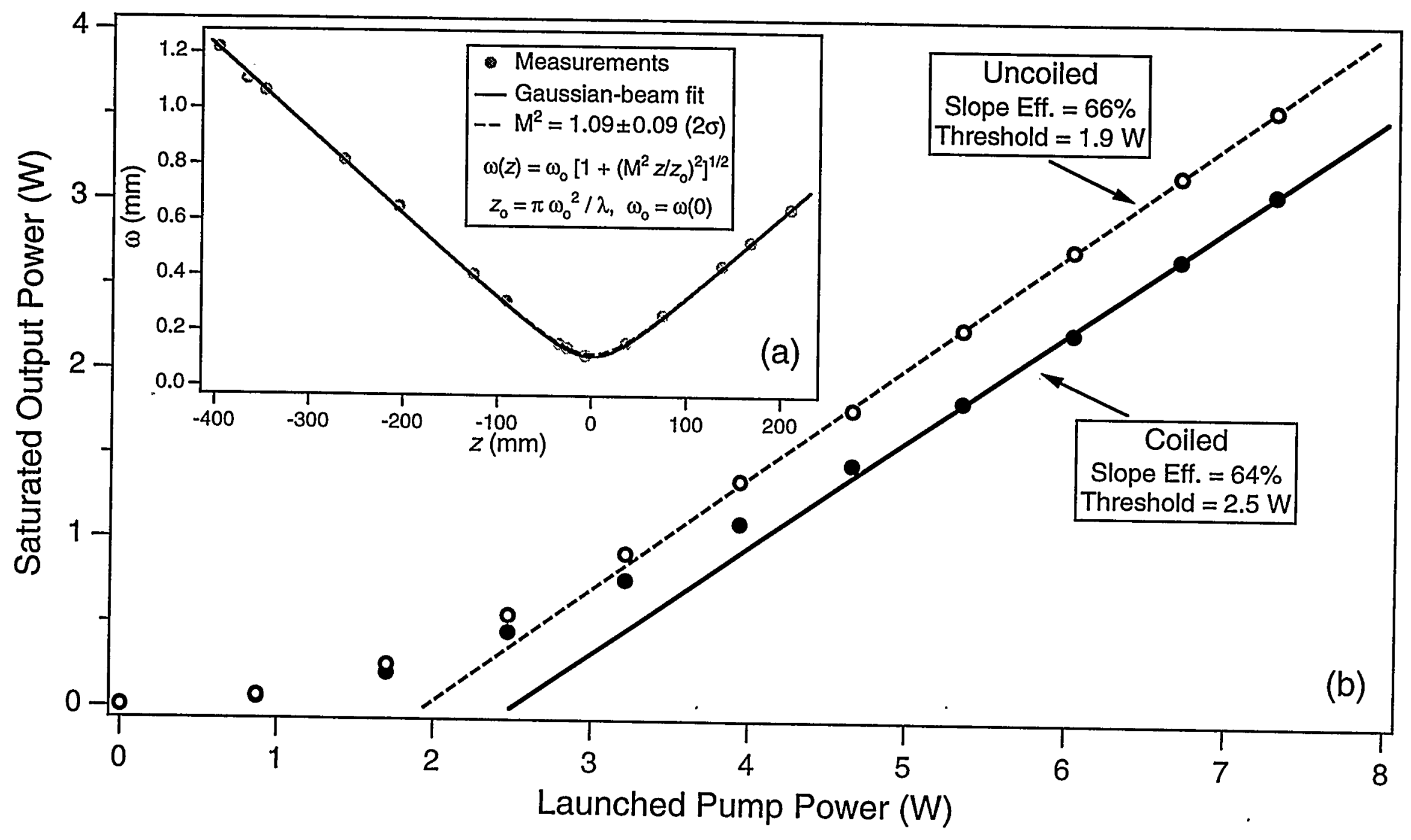

